\title{
Special issue on emerging multimedia applications
}

\author{
Han-Chieh Chao • Sajid Hussain • Shu-Ching Chen • \\ William I. Grosky
}

Published online: 18 December 2009

(C) Springer Science+Business Media, LLC 2009

Due to the latest developments in electronics industry, the emerging multimedia applications are smart, efficient as well as sensitive to the deployment constraints. The intelligent and soft computing techniques provide scalable and energy efficient multimedia solutions. The emerging multimedia tools can be used for various pervasive and ubiquitous applications such as health care, environmental monitoring, security and surveillance. This special issue includes 12 papers relating to these emerging applications.

In the paper titled "Stereo Panorama Acquisition and Display for Virtual Tour Systems," Huang and Klette investigate methods for determining optimum parameters both for highaccuracy stereo panoramic image recording and displaying. They focus on automatic image disparity enhancement while displaying a stereo panorama. The experimental results show that these parameters are critical for ensuring high quality stereo viewing. The derived formulas are applicable in technologies for stereo panorama imaging or stereoscopic displaying.

Xiao et al. present "A Group of Novel Approaches and a Toolkit for Motion Capture Data Reusing”. They propose approaches for posture feature representation, motion sequence segmentation, key-frame extraction and content-based motion retrieval, which are very important for Motion Capture (MoCap) data reusing.

In paper titled "A Portable Toolkit for Supporting End-User Personalization and Control in Context-Aware Applications", Kawsar et al. address the personalization and control issues at the system interface level in parallel to context centric design. They present a toolkit that provides support for extending context-aware applications with end-user personalization and control features. The toolkit can be used with various existing

H.-C. Chao

Department of Electronic Engineering, National Ilan University, I-lan, Taiwan, ROC e-mail: hcc@niu.edu.tw

S. Hussain

Department of Mathematics and Computer Science, Fisk University, Nashville, Tennessee, USA e-mail: shussain@fisk.edu

S.-C. Chen $(\bowtie)$

School of Computing and Information Sciences, Florida International University, Miami, FL, USA e-mail: chens@cs.fiu.edu

W. I. Grosky

Department of Computer and Information Science, University of Michigan-Dearborn, Dearborn, Michigan, USA

e-mail: wgrosky@umich.edu 
middleware. Further, the existing context-aware applications can be augmented to provide end-user personalization and control support.

Han et al. provide "Music Emotion Classification and Context-based Music Recommendation". They propose a novel emotion state transition model (ESTM) to model human emotional states and their transitions by music. They also present context-based music recommendation (COMUS) ontology for modeling user's musical preferences and context, and for supporting reasoning about the user's desired emotion and preferences. For mapping low-level features to ESTM, they collected various high-dimensional music feature data and applied nonnegative matrix factorization (NMF) for their dimension reduction.

In paper titled "A New Unequal Error Protection Scheme Based on FMO," Shih and Tsai present a novel scheme for video transmission over error-prone networks. The proposed scheme is based on the error resilient features of H.264/AVC and employs an unequal error protection (UEP) approach to protect effectively the streams. They classify macro blocks into slice groups using the explicit mode of Flexible Macroblock Ordering (FMO). Further, a Converged Motion Estimation (CME) is used to improve the proposed UEP scheme.

Hakkoymaz presents "Multimedia Presentation Organization and Playout Management using Intelligent Agents". A model is proposed for organization, computation and management of automated multimedia presentations based on active multimedia segments retrieved from a multimedia information system and the Web. Based on event-point representation, three methods are given for playing out the constructed presentations at the presentation terminal.

In paper entitled "An Automatic Feature Generation Approach to Multiple Instance Learning and Its Applications to Image Databases", Cheng et al. propose a novel learning technique which transforms the MIL problem into a standard supervised learning problem by defining a feature vector for each image bag. With this new image representation, standard supervised learning algorithms can be applied to classify the images into the predefined categories.

Kovacevic et al. present "Adaptive Content-Based Music Retrieval System". It is a tunable content-based music retrieval (CBMR) system, which is suitable for retrieval of music audio clips. The audio clips are represented as extracted feature vectors. It improves the retrieval performance genetic algorithm based tuning process. For efficient retrieval of audio clips, the R-tree index is based on the clustering of feature vectors. The proposed CBMR system implements the point query and the n-nearest neighbors query with the $\mathrm{O}$ ( $\log n$ ) time complexity. Different objective functions based on cluster similarity and dissimilarity measures are used for the genetic algorithm.

Kolekar and Sengupta investigate "Semantic Concept Mining in Cricket Videos for Automated Highlight Generation." They automate highlight generation of broadcast sports video sequences from its extracted events and semantic concepts. The proposed method extracts event sequence from video and classifies each sequence into a concept by sequential association mining.

In paper entitled, "A Multimedia Application for Watermarking Digital Images Based on a Content Based Image Retrieval Technique", Tsolis et al. investigate the implementation of a robust multimedia application for watermarking digital images. The proposed technique is based on an innovative spread spectrum analysis algorithm for watermark embedding and on a content-based image retrieval technique for watermark detection. It combines the advantages of both "detectable" and "readable" watermarks.

Batko et al. present "Building a Web-scale Image Similarity Search System". They investigate a similarity search system on a test collection of more than 50 million images. 
They address the challenge of obtaining a collection of images of this scale with the corresponding descriptive features. They also develop scalable indexing and searching mechanisms and answer similarity queries in real-time.

In paper entitled "Simulated Evaluation of Faceted Browsing based on Feature Selection", Hopfgartner et al. explore the limitations of facet based browsing which uses sub-needs of an information need for querying and organizing the search process in video retrieval. They reduce the dimensionality of features by selecting the most important ones. Facets created by users are simulated by clustering video shots using textual and visual features.

Finally, we would like to thank our reviewers, the organizers of the Second International Conference on Multimedia and Ubiquitous Engineering (MUE), and the editorial staff of JUCS journal.

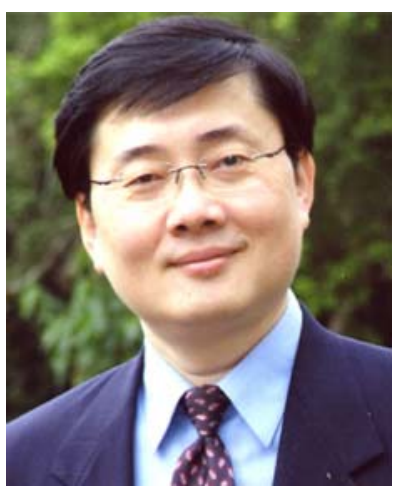

Han-Chieh Chao is currently a jointly appointed professor in the institute of Computer Science and Information Engineering and the institute and department of electronic engineering at National Ilan University, Taiwan; jointly adjunct professor in the institute and department of electrical engineering at National Dong Hwa University, Taiwan; and honorary adjunct professor in Beijing Jiaotong University (985 University), Xiamen University (985 University), Lanzhou University (985 University) and Yantai University, China. His research interests include High Speed Networks, Wireless Networks, IPv6 based Networks, Digital Creative Arts and Digital Divide. He received his MS and Ph.D. degrees in Electrical Engineering from Purdue University in 1989 and 1993 respectively. He has authored or co-authored 4 books and has published about 250 refereed professional research papers. He has completed 90 MSEE and $2 \mathrm{PhD}$ thesis students. Dr. Chao has received many research awards, including Purdue University SRC awards, and NSC research awards (National Science Council of Taiwan). He also received many funded research grants from NSC, Ministry of Education (MOE), RDEC, Industrial Technology of Research Institute, Institute of Information Industry and FarEasTone Telecommunications Lab. Dr. Chao has been invited frequently to give talks at national and international conferences and research organizations. Dr. Chao is also serving as an IPv6 Steering Committee member and co-chair of R\&D division of the NICI (National Information and Communication Initiative, a ministry level government agency which aims to integrate domestic IT and Telecom projects of Taiwan), Co-chair of the Technical Area for IPv6 Forum Taiwan, the executive editor of the Journal of Internet Technology and the Editor-in-Chief for International Journal of Internet Protocol Technology and International Journal of Ad Hoc and Ubiquitous Computing. Dr. Chao has served as the guest editors for Mobile Networking and Applications (ACM MONET), IEEE JSAC, IEEE Communications Magazine, Computer Communications, IEE Proceedings Communications, and Wireless Communications \& Mobile Computing. Dr. Chao is an IEEE senior member, a Fellow of the Institution of Engineering and Technology (FIET), and Chartered Fellow of British Computer Society (FBCS). 


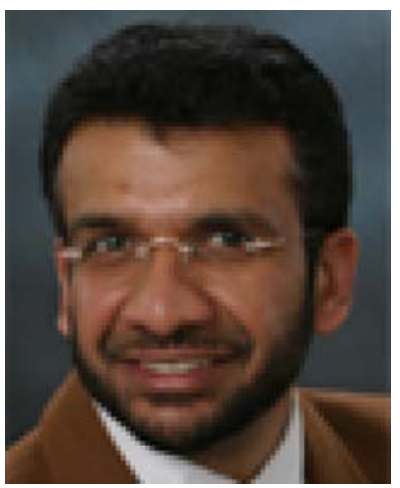

Dr. Sajid Hussain is Associate Professor in the Computer Science Department at Fisk University, Nashville, USA. He worked as Associate Professor and Assistant Professor in the Jodrey School of Computer Science, Acadia University, Canada, 2005-09. He received Ph.D. in Electrical Engineering from the University of Manitoba, Canada, 2004. Further, he holds Adjunct Professor positions in the Faculty of Computer Science at Dalhousie University and Jodrey School of Computer Science at Acdia University, as well as a Visiting Lecturer position for SPIE.

Dr. Hussain is investigating secure and energy efficient communication protocols in sensor networks and MANETS for mobile, ubiquitous, and pervasive applications. He is interested in smart homes, telehealth, and industry automation. He has published more than 70 refereed journal, conference, and workshop papers. His research is financially supported by several grants and contracts, such as NSERC Canada, NRC Canada, AIF/ACOA, and NSHRF.

He has co-organized several journal special issues, conferences, and workshops. He has served on many technical program committees and reviewed papers for several journals. Further, he has reviewed grant proposals for NSERC's Discovery, SPG, and RTI grants. He is a member of IEEE, ACM, IET, SPIE, CIPS, CAIAC, and Sigma Xi societies.

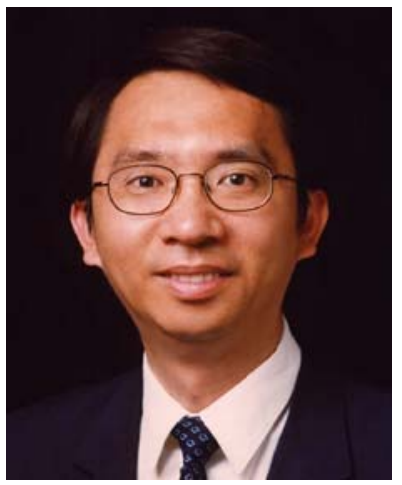

Shu-Ching Chen is a Full Professor in the School of Computing and Information Sciences (SCIS), Florida International University (FIU), Miami since August 2009. Prior to that, he was an Assistant/Associate Professor in SCIS at FIU from 1999. He received Master's degrees in Computer Science, Electrical Engineering, and Civil Engineering in 1992, 1995, and 1996, respectively, and the Ph.D. degree in Electrical and Computer Engineering in 1998, all from Purdue University, West Lafayette, IN, USA. His main research interests include distributed multimedia database management systems and multimedia data mining.

Dr. Chen has authored or coauthored more than 200 research papers. Dr. Chen received the best paper award from the 2006 IEEE International Symposium on Multimedia. He was awarded the IEEE Systems, Man, and Cybernetics (SMC) Society's Outstanding Contribution Award in 2005 and was co-recipient, along 
with Dr. Rubin, of the IEEE Most Active SMC Technical Committee Award in 2006. He was also awarded the Inaugural Excellence in Graduate Mentorship Award from FIU in 2006, the University Outstanding Faculty Research Award from FIU in 2004, the Outstanding Faculty Service Award from SCIS in 2004, and the Outstanding Faculty Research Award from SCIS in 2002. He has been a General Chair and Program Chair for more than 28 conferences, symposiums, and workshops. He was a member of a steering committee and several panels. He also serves/served as a member of technical program committee for more than 100 professional meetings. He is the Editor-in-Chief of International Journal of Multimedia Data Engineering and Management and Associate Editors/Editorial Board for several journals. Dr. Chen also has been a guest editor for more than ten journal special issues.

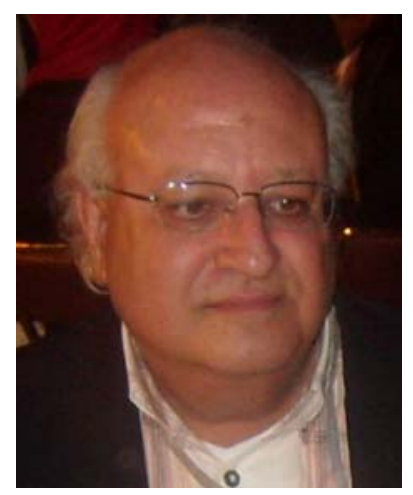

William I. Grosky is currently professor and chair of the Department of Computer and Information Science at the University of Michigan-Dearborn. Before joining UMD in 2001, he was professor and chair of the Department of Computer Science at Wayne State University, as well as an assistant professor of Information and Computer Science at the Georgia Institute of Technology in Atlanta. His current research interests are in multimedia information systems, databases, and the semantic web. He has given many short courses in the area of database management for local industries and has been invited to lecture on multimedia information systems world-wide. Serving also on many database and multimedia conference program committees, he was an Editor-in-Chief of IEEE Multimedia, and is currently on the editorial boards of several international journals, including IEEE Multimedia, International Journal on Semantic Web and Information Systems, and International Journal of Semantic Computing. 